\title{
Avaliando Resultados de um Programa de Transferência de Renda: o Impacto do Bolsa-Escola sobre os Gastos das Famílias Brasileiras
}

\author{
- Anne Caroline Costa Resende* - Ana Maria Hermeto Camilo de Oliveira **
}

\begin{abstract}
RESUMO
Este trabalho tem como objetivo analisar os efeitos de programas de transferências monetárias sobre os gastos totais bem como sobre seus componentes para as famílias beneficiárias no Brasil, através do programa Bolsa-Escola. Para se alcançar tal objetivo é adotado um método não- experimental conhecido como pareamento baseado no escore de propensão (propensity score matching), utilizando os microdados da Pesquisa de Orçamentos Familiares (POF) 2002-2003. Realiza-se, ainda, uma análise de sensibilidade dos resultados obtidos a fim de se verificar a robustez do método à presença de variáveis não observadas, bem como à especificação do modelo de escore de propensão. Os resultados estimados sugerem um efeito positivo das transferências monetárias sobre o consumo das famílias pobres beneficiárias. $O$ fato de os recursos serem prioritariamente destinados a despesas com alimentos, educação, produtos de higiene e vestuário em detrimento de itens como bebidas e cigarros, despesas diversas e bens duráveis significa que, em alguma medida, estes recursos estão sendo gastos de forma eficiente. É provável, também, que o aumento do consumo destas famílias eleve o seu nível de bem-estar, representando um "alívio" imediato sobre a pobreza.
\end{abstract}

Palavras-Chave

Programas Condicionais de Transferências, escore de propensão, gastos

\section{ABSTRACT}

The aim of this work is to analyze the effects of cash transfers of public programs on household's expenditures and their components, taking the Brazilian program Bolsa-Escola as a representative example. It is used a non experimental method called propensity score matching, with microdata from the Pesquisa de Orçamentos Familiares (POF) 2002-2003. Further, a sensitivity analysis is carried to certify that the propensity score model is well specified and still works in the presence of unobservable variables. Results suggest a positive effect of cash transfers on the poor beneficiary household's consumption. The fact that household's expenditures are concentrated on education, hygiene products, clothing and general food instead of drinks, cigarettes and durable goods means that, at some level, the money transferred is being efficiently spent. Hence, the increased consumption of the observed households enhances their welfare, which means an immediate relief from poverty conditions.

\section{KEYWORDS}

Conditional Cash Transfer Programs, propensity score matching, expenditures

\section{JEL CLASSIFICATION}

I38, $\mathrm{Cl} 4$

\footnotetext{
* Doutoranda na Royal Holloway - University of London. E-mail: A.C.C.Resende@rhul.ac.uk.

** Professora Adjunta do Departamento de Ciências Econômicas da UFMG e do CEDEPLAR. Endereço para contato: Faculdade de Ciências Econômicas - Av. Antônio Carlos, 6627. CEP: 31270-90I, Belo Horizonte, MG. E-mail: ahermeto@cedeplar.ufmg.br.

(Recebido em janeiro de 2007. Aceito para publicação em agosto de 2007).
} 


\section{INTRODUÇÃO}

Programas de transferência condicionada de renda são políticas sociais cada vez mais empregadas no combate à pobreza em países em desenvolvimento. Estes programas têm como finalidade prover assistência no curto prazo para as famílias pobres e assim atenuar a pobreza corrente. Ao mesmo tempo, propiciam o investimento no desenvolvimento de capital humano através das condicionalidades do recebimento do benefício, combatendo, no longo prazo, a pobreza futura. Outros objetivos menos explícitos, mas não menos importantes, incluem a melhoria do bem- estar das famílias, inclusive a redistribuição de renda e a promoção da inclusão social. Estudos recentes (ROCHA, 2004; SOARES, 2006; FERREIRA; LEITE; LITCHFIELD, 2006) evidenciam os potenciais efeitos dos programas de transferência sobre a redução das desigualdades e da pobreza no País, destacando a importância deste tipo de política.

Uma questão emerge em relação à eficácia destes programas: como verificar o impacto de um programa social sobre o bem-estar das famílias? Esta é uma questão ampla que pode ser respondida sob várias perspectivas. Pode-se avaliar o impacto de um determinado programa analisando o cumprimento das condicionalidades exigidas, como por exemplo, o aumento da freqüência à escola. Do mesmo modo, pode-se verificar os efeitos sobre o número de consultas pré-natais para as mães beneficiárias. No entanto, a despeito dos objetivos diretos, estes programas também apresentam efeitos não esperados, não explícitos como objetivos. A mensuração de tais efeitos, por sua vez, também permite fazer inferências a respeito do nível de bem-estar destas famílias. Dentre os efeitos não esperados destacam-se os impactos das transferências monetárias sobre os gastos e, portanto, sobre o consumo das famílias beneficiárias.

Uma primeira geração de avaliações de programas condicionais de transferência de renda encontrou efeitos positivos sobre as taxas de matrícula, freqüência à escola e redução no trabalho infantil para as crianças brasileiras. Outros países apresentam, adicionalmente, avaliações sobre os níveis de consumo das famílias. Para o Brasil há basicamente dois estudos que realizam esse tipo de avaliação: o primeiro sobre o Bolsa-Alimentação - que se concentra, no entanto, somente no consumo de alimentos - e o segundo, realizado por Vaz e Assunção (2005), através da utilização de uma regressão padrão. Tais estudos verificam que as famílias beneficiárias do programa Bolsa-Escola tendem a gastar mais com alimentos e não reduzem seus gastos em educação. Neste sentido, há uma lacuna de estudos deste tipo, sobretudo dada a disponibilidade de microdados que permitem explorar metodologias de avaliação em relação a uma vasta gama de resultados.

Deste modo, a finalidade deste trabalho é analisar os impactos das transferências monetárias advindos de programas sociais de transferências de renda, em geral, sobre os 
gastos das famílias beneficiárias e, portanto, sobre o bem-estar destas e de suas crianças. Contudo, tal análise será realizada através da aplicação específica do programa Bolsa-Escola (B.E.), supondo que este seja um exemplo representativo de programa de transferência condicional de renda.

Além dos impactos sobre os gastos totais, será realizada uma análise sobre os componentes do consumo (alimentação, habitação, vestuário, educação, saúde e demais despesas). A avaliação dos efeitos sobre cada componente dos gastos e sobre itens específicos permitirá verificar como as famílias beneficiárias alocam os recursos advindos do programa, e se há uma apropriação desproporcional dos benefícios por parte dos adultos.

Em geral, espera-se que as transferências recebidas do programa tenham um efeito positivo sobre as despesas de consumo, dado que tais transferências aumentam a renda disponível das famílias. Attanasio e Mesnard (2005) argumentam, no entanto, que este efeito não é tão imediato quanto parece. Em primeiro lugar, a renda disponível não irá aumentar necessariamente no mesmo montante das transferências recebidas, uma vez que as condições impostas pelo programa podem reduzir outras formas de rendimentos, como por exemplo, a renda do trabalho infantil. Segundo, os valores recebidos podem não ser inteiramente gastos para o consumo de bens, já que as famílias podem decidir poupar uma fração, utilizá-los para o pagamento de débitos correntes ou no investimento de atividades produtivas.

Entende-se que o aumento dos gastos e, conseqüentemente, do consumo, tenha por finalidade atenuar as adversidades das famílias mais pobres. O bem-estar destas famílias pode ser mensurado através do "alívio" imediato em termos de consumo, e, assim, sobre as condições adversas que estas enfrentam. Segundo Attanasio et al. (2005), existem diversas razões pelas quais os programas condicionados de transferências podem não obter os efeitos desejados, tais como: o fato de o programa existir não significa que as famílias-alvo irão participar; o custo de se monitorar o comprimento das condicionalidades pode ser relativamente superior aos valores das transferências. A despeito da existência da condicionalidade, a transferência de recursos monetários para as famílias pobres não significa necessariamente que estes serão gastos da maneira ambicionada. As famílias podem utilizar parte destes recursos para o consumo de tabaco, bebidas alcoólicas e outros bens destinados a adultos, que geralmente são considerados indesejáveis. $\mathrm{Ou}$, de forma similar, podem destinar a maior parte dos recursos a outros membros das famílias em detrimento das crianças. 
Programas de transferência condicionada de renda, cujo objetivo prioritário é a redução das desigualdades, são políticas sociais cada vez mais empregadas no combate à pobreza em países em desenvolvimento. Agrupados sob a sigla "MISA" - Minimum Income for School Attendance - estes programas têm como finalidade atenuar a pobreza corrente e combater a pobreza futura condicionando as transferências à participação no sistema formal de ensino; alguns apresentam, ainda, outras condicionalidades geralmente associadas a saúde e alimentação. Tais programas são também conhecidos como programas condicionais de transferências de renda (ou Conditional Cash Transfers (CCT)).

Os chamados CCT's vêm se tornando ferramentas amplamente utilizadas com a finalidade de se obter melhorias nos status de educação, saúde e nutrição das crianças pobres. Alguns exemplos destes são o mexicano Progresa (Programa Nacional de Educación, Salud y Alimentación), em vigor desde 1997; na Nicarágua, o programa Red de Proteción Social (RPS), atuando desde 2000; na Colômbia, o Familias em Acción que teve início em 2001; e o indiano Food-for-Education (FFE) que iniciou em 1995, entre outros. ${ }^{1}$

No Brasil, o Bolsa-Escola foi um dos precursores destes programas. Adotado inicialmente no âmbito municipal, o Bolsa-Escola teve início no primeiro semestre de 1995 com a experiência pioneira do município de Campinas (SP), seguido pelo Distrito Federal e por outros municípios. Entretanto, as várias tentativas de implementação por iniciativa municipal passaram a demonstrar a necessidade de federalização do programa, uma vez que a grande maioria dos municípios não possuía capacidade financeira e operacional para garantir o seu funcionamento. Devido a esse fato, em 2001, o governo federal passou a arcar com as responsabilidades de financiamento, gestão e operacionalização do Bolsa-Escola (ROCHA, 2005).

O foco do programa Bolsa-Escola são as famílias pobres e com crianças em idade escolar. Sua população-alvo constitui-se de famílias com renda mensal per capita de até R \$ 90,00 que possuem crianças entre 6 e 15 anos de idade. O benefício mensal por criança é de $\mathrm{R} \$ 15,00$ até o máximo de $\mathrm{R} \$ 45,00$ por família e o seu recebimento está condicionado ao fato de todas as crianças em idade escolar estarem matriculadas e freqüentando a escola. O percentual mínimo de freqüência exigida é de $85 \%$ e as escolas ficariam encarregadas de reportar tais números aos governos municipais beneficiários do programa.

1 Para uma apresentação dos resultados de avaliações de programas nacionais e internacionais, ver Rawlings e Rubio (2003) e Das, Do e Özler (2004). 


\section{METODOLOGLA}

O termo avaliação refere-se à mensuração do impacto de intervenções, tais como a participação em um programa de treinamento ou o recebimento de uma transferência de renda de um programa social, sobre os efeitos de interesse. $\mathrm{O}$ termo efeito referese a mudanças no status das variáveis relevantes. O problema central na avaliação de impacto é a inferência de uma conexão causal entre o tratamento (a participação em um determinado programa) e o efeito (CAMERON; TRIVEDI, 2005). A relevância das avaliações de impacto é direta, pois seus efeitos podem ser associados a programas sociais ou melhorias em programas existentes a fim de se atingir os objetivos da política social.

\subsection{Matching - Um Estimador Econométrico para Avaliações}

Para as finalidades deste trabalho, será utilizada a metodologia conhecida como matching ou pareamento. O pareamento é um método amplamente utilizado na literatura de avaliação, tornando-se popular na estimativa de efeitos causais de tratamento. No presente estudo, tal método será empregado para avaliar os efeitos das transferências do programa Bolsa-Escola sobre os gastos das famílias beneficiárias, bem como sobre os componentes do consumo ${ }^{2}$, tais como alimentos, habitação, vestuário, educação, saúde e demais despesas.

O objetivo do pareamento é encontrar um grupo de comparação ideal em relação ao grupo de tratamento a partir de uma amostra de não-participantes. Em seguida, estimam-se os efeitos do tratamento por meio da diferença entre os resultados médios dos grupos. O grupo de comparação é emparelhado ao grupo de tratamento através de uma série de características observáveis ou por meio do propensity score (escore de propensão ou probabilidade predita de participação).

Utilizando a terminologia de Heckman, Ichimura e Todd (1997), representamos o status de tratamento de um indivíduo através de uma variável dummy $\mathrm{D}$ que possui valor igual a $\mathrm{l}$, se o indivíduo participa do programa, e valor 0 , caso ele não participe. Representemos ainda por $Y_{1 i} \mathrm{o}$ valor da variável de interesse para o indivíduo $i$, caso ele esteja sujeito ao tratamento (1), e $Y_{0 i}$ o valor da mesma variável, caso este indivíduo esteja exposto ao controle (0).

O impacto médio do treinamento sobre os participantes será:

2 Os termos gasto e consumo referem-se às despesas monetárias realizadas pelas famílias e são utilizados de forma equivalente neste trabalho. 


$$
\Pi_{i}=E\left[Y_{l i}-Y_{0 i} \mid D_{i}=1\right]=E\left(Y_{l i} \mid D_{i}=1\right)-E\left(Y_{0 i} \mid D_{i}=1\right)
$$

Na literatura de avaliação, $E\left[Y_{l i}-Y_{0 i} \mid D_{i}=1\right]$ é chamado de efeito do tratamento ou efeito médio do tratamento sobre o tratado (average treatment effect on treated (ATT)).

O problema é que o resultado contrafactual de um indivíduo sob tratamento $E\left(Y_{0 i} \mid D_{i}\right.$ =1) (2) não pode ser observado, uma vez que um indivíduo só pode ser tratamento ou controle em um ponto específico do tempo. Conseqüentemente, devemos impor certas hipóteses em (l) a fim de que o ATT possa ser estimado. Uma forma é substituir o resultado esperado do indivíduo que participou se ele não tivesse participado (2), com o resultado esperado dos indivíduos que de fato não participaram $E\left(Y_{0 i} \mid D_{i}=0\right)$ (3) . Entretanto, como a escolha dos participantes no programa não foi conduzida aleatoriamente, não podemos assumir que substituindo (2) por (3) teremos uma estimativa não viesada, porque é improvável que $E\left(Y_{0_{i}} \mid D_{i}=1\right)=E\left(Y_{0_{i}} \mid D_{i}=0\right)$. Tal improbabilidade deve-se à existência de viés, que surge devido a diferenças nas características observáveis e a diferenças nos atributos não-observáveis entre os grupos de tratamento e controle. Ao levarmos em consideração as características observáveis do processo de seleção bem como as características que potencialmente influenciam o resultado de interesse nos indivíduos tratados, podemos reescrever a última equação como:

$$
E\left(Y_{l i} Y_{0 i} \mid D_{i}=1, X\right)=E\left(Y_{l i} \mid D_{i}=1, X\right)-E\left(Y_{0 i} \mid D_{i}=0, X\right)
$$

em que $X$ representa um vetor das características observáveis. De acordo com a hipótese de identificação geralmente adotada, o processo de seleção ocorre segundo características observáveis, tal que as pessoas com tais características idênticas possuem a mesma probabilidade de serem alocadas como tratamento ou controle. Isto significa que:

$$
\left(Y_{0 i}, Y_{1 i} \perp D_{i} \mid X\right) \text { e } E\left(Y_{0 i} \mid X_{i}, D_{i}=1\right)=E\left(Y_{0 i} \mid X_{i}, D_{i}=0\right)^{3}
$$

em que $\perp$ denota independência, significando que os resultados potenciais independem da participação do programa dadas as características observáveis $X$ - esta hipótese é conhecida como Hipótese da Independência Condicional ou Conditional Independence Assumption (CIA). ${ }^{4}$

O problema de utilizarmos tal método é que quanto mais características são utilizadas como base, mais difícil será encontrar unidades no grupo de controle que tenham exa-

3 Para mais detalhes, ver Hirano, Imbens e Ridder (2000).

4 Esta hipótese implica que a seleção é baseada somente nas características observáveis e que todas as variáveis que influenciam a participação no programa e os resultados potenciais são simultaneamente observados pelo pesquisador. Claramente, esta é uma hipótese forte. Neste trabalho, assume-se que esta condição seja verdadeira. 
tamente as mesmas características que as definidas no grupo de tratamento, o que se torna ainda mais complexo quando as variáveis observáveis são variáveis contínuas.

\subsection{O Escore de Propensão}

Pelo fato de a participação não ter sido desenhada de forma aleatória, segundo Attanasio et al. (2004), uma simples comparação entre os participantes e não-participantes do programa poderia ser bastante equivocada por duas razões. Primeiro, diferenças ex-post nos resultados poderiam refletir simplesmente diferenças pré-programa. Segundo, o efeito do programa pode ser uma função de variáveis de background (escolaridade do chefe, número de crianças etc) que podem ser diferentes entre os grupos de tratamento e controle. Estes problemas podem ser solucionados utilizando-se o método do escore de propensão.

Para lidar com o problema da dimensionalidade do pareamento, Rosenbaum e Rubin (1983) desenvolveram um método conhecido como Propensity Score Matching. Estes autores mostraram que tal método pode ser implementado através de uma única variável de controle, o escore de propensão. O escore de propensão $P(x)$ é definido como a probabilidade condicional de um indivíduo receber o tratamento dado suas características observáveis $X$. Isto é,

$$
P(X)=\text { Probabilidade }(D=1 \mid X) .{ }^{5}
$$

Rosenbaum e Rubin (1983) mostram que na expressão (4), $X$ pode ser substituído por $P(X)$, assim:

$$
E\left(Y_{1}, Y_{0} \mid D=1, P(X)\right)=E\left(Y_{1} \mid D=1, P(X)\right)-E\left(Y_{0} \mid D=0, P(X)\right)
$$

Se o tratamento e os resultados esperados são independentes condicionais às variáveis de pré-tratamento, estes também serão independentes condicionais à probabilidade de receber o tratamento dadas as características observáveis, isto é, condicional ao escore de propensão. ${ }^{6}$

$$
\left(Y_{0}, Y_{1} \perp D \mid P(X)\right)
$$

A utilização do escore de propensão baseia-se em duas hipóteses-chave. Primeiro, como já mencionado, a seleção nos observáveis requer que a participação no programa seja independente dos resultados, condicional nas co-variáveis. A segunda hipótese

5 O método do escore de propensão e o efeito do tratamento serão calculados a partir de algumas funções do STATA que foram desenvolvidas recentemente por Becker e Ichino (2002).

6 Ver Rosenbaum e Rubin (1983) ou Imbens (2000) para a prova. 
refere-se à existência de um suporte comum. Esta condição requer que existam unidades de ambos os grupos, tratamento e controle, para cada característica $X$ para a qual deseja-se comparar.

$$
0<P(X)<1
$$

Isto assegura que para cada indivíduo tratado exista outro indivíduo não tratado pareado, com valores similares de $X$ (HECKMAN; LALONDE; SMITH, 1999).

\subsection{Consideraçôes a Respeito do Viés}

Admitindo que:

$$
C=E\left(Y_{l i} \mid D_{i}=1\right)-E\left(Y_{0 i} \mid D_{i}=0\right)
$$

A identidade associando (1) e (4) será:

$$
(4)=(1)+V
$$

Em que o $V$ é o viés na estimativa, dado por:

$$
V=E\left(Y_{0 i} \mid D_{i}=1\right)-E\left(Y_{0 i} \mid D_{i}=0\right)
$$

Assim, o viés surge devido à utilização dos resultados médios do grupo de comparação como proxy para os resultados médios dos participantes do programa caso eles não tivessem participado.

De acordo com Heckman, Ichimura e Todd (1997), o viés pode ser dividido em três componentes básicos: o primeiro componente surge devido à falta de suporte comum. ${ }^{7} \mathrm{O}$ segundo surge devido ao viés nos observáveis enquanto o terceiro deve-se às diferenças nos resultados que permanecem mesmo após levarmos em consideração as características observáveis e realizarmos as comparações em uma região de suporte comum. Este componente deve-se a diferenças nos não- observáveis, conhecido como viés de seleção. Este viés surge quando para dados valores de $X$ há uma relação sistemática entre a participação no programa e os resultados, ou seja, há variáveis não observadas que conjuntamente influenciam os resultados e a participação no programa, condicionais às variáveis observáveis. Para lidar com o viés a melhor maneira seria alocar o programa aleatoriamente, pois dessa forma poderíamos garantir que

7 De acordo com Heckman, Ichimura e Todd (1997), violar a condição de suporte comum é a principal fonte de viés na avaliação. 
participantes e não-participantes teriam o mesmo resultado esperado na ausência do programa. $^{8}$

Uma importante observação a ser feita a respeito do método de pareamento é que este elimina duas das três fontes de viés. O primeiro componente é eliminado através da realização do pareamento em uma região de suporte comum. O pareamento cuidadoso do grupo de comparação baseado nas características observáveis elimina o segundo componente de viés. Contudo, os métodos de pareamento somente lidam com características observáveis, restando o problema de heterogeneidade latente que leva a um possível viés na estimação do impacto do programa.

Portanto, deve-se ter em mente que o método do escore de propensão permite reduzir, mas não eliminar, o viés gerado pelos fatores não-observáveis. A extensão na qual o viés é reduzido depende crucialmente da riqueza e da qualidade das variáveis de controle em que o escore de propensão é computado e o pareamento é realizado (BECKER; ICHINO, 2002).

\subsection{Calculando o ATT Utilizando Estimadores Não-Experimentais Baseados no Escore de Propensão ${ }^{9}$}

A estimativa do escore de propensão não é suficiente para estimar o efeito médio do tratamento. A causa disso é que a probabilidade de encontrarmos dois indivíduos com exatamente o mesmo valor de escore de propensão é em princípio zero, uma vez que $p(X)$ é uma variável contínua. Vários métodos foram propostos na literatura para lidar com este problema; neste trabalho, utilizaremos os estimadores mais comumente empregados.

Dentre os diversos algoritmos de pareamento empregados na literatura optou-se pela utilização do pareamento estratificado (stratification matching (SM)). ${ }^{10}$ Tal decisão decorre do fato de que a estimativa do ATT e do número final de blocos gerados no escore de propensão, são baseados no princípio da estratificação da amostra. ${ }^{11}$

O método do pareamento estratificado consiste em dividir a variação dos escores de propensão em intervalos tal que em cada um destes as unidades tratadas e as unidades

8 A randomização possui a vantagem-chave em relação aos métodos não experimentais de gerar um grupo de controle que possui a mesma distribuição das características observáveis e não-observáveis que as do grupo de tratamento. Entretanto, como já mencionado, a participação no programa não foi conduzida aleatoriamente, mas seguiu certos processos de seleção.

9 Esta sessão baseia-se em Becker e Ichino (2002).

10 A estimativa do ATT será realizada considerando o uso do suporte comum para todas as observações.

11 Para uma apresentação dos demais estimadores baseados no escore de propensão usualmente empregado, bem como para uma comparação metodológica entre estes, ver Resende (2006). 
de controle possuam na média o mesmo escore de propensão. Em seguida, em cada intervalo é calculada a diferença dos resultados médios entre os grupos de participantes e não-participantes. O ATT é finalmente obtido através da média ponderada dessas diferenças, com os pesos sendo determinados pela distribuição das unidades tratadas entre os blocos. No SM as observações nos blocos que não possuem tratamento ou controle são descartadas. Se $q$ representa o índice dos blocos definidos no intervalo do escore de propensão, dentro de cada bloco é computado:

$$
T_{q}^{S}=\frac{\sum_{i \in I(q)} Y_{i}^{T}}{N_{q}^{T}}-\frac{\sum_{j \in I(q)} Y_{j}^{C}}{N_{q}^{C}}
$$

onde $I_{(q)}$ representa o conjunto das unidades no bloco $q$ enquanto $N_{q}^{T}$ e $N_{q}^{C}$ representam as quantidades de unidades tratadas e de controle no bloco $q$.

\subsection{Análise de Sensibilidade Utilizando o Método de Limites de Rosenbaum ${ }^{12}$}

Como discutido anteriormente, os estimadores baseados no escore de propensão não são estimadores consistentes do efeito do tratamento se a participação no programa é endógena, isto é, se variáveis não observadas que afetam o processo de seleção também afetam os resultados. Fatores não observados, como por exemplo a motivação, provavelmente se correlacionam com $D$ e $Y$. Portanto, a omissão de tal característica individual pode enviesar os resultados do efeito médio do tratamento sobre o tratado estimado sob a hipótese de exogeneidade.

Visto que não é possível estimar a magnitude do viés de seleção em dados não experimentais, uma importante ferramenta pode ser empregada para avaliá-lo na estimação dos efeitos causais. A chamada "análise de limites" (bounds analysis), que se popularizou nos anos recentes, avalia o impacto potencial do viés de seleção que surge devido a variáveis não observadas. Neste estudo será utilizado o método conhecido como Rosenbaum bounds (ROSENBAUM, 2002; DIPRETE; GANGL, 2004), que por sua vez permite determinar quão "forte" deve ser a influência de uma variável omitida sobre a seleção na participação a fim de prejudicar as conclusões a respeito dos efeitos causais, obtidos com as análises de pareamento. ${ }^{13}$

A análise de sensibilidade pode ser utilizada para testar a robustez dos resultados à presença de viés devido a uma co-variável omitida. O objetivo desta análise no presen-

12 Para uma discussão sobre análise de sensibilidade utilizando o método das variáveis instrumentais, ver Diprete e Gangl (2004).

13 Este método será esboçado brevemente; uma discussão extensiva pode ser encontrada em Rosenbaum (2002). 
te trabalho é avaliar como uma variável não-observável que afeta tanto a participação no programa Bolsa-Escola como os resultados (impacto sobre o consumo) podem alterar as conclusões a respeito do efeito do tratamento.

Considerando-se que a probabilidade de participação de um indivíduo $i$ seja dada por: ${ }^{14}$

$$
\pi_{i}=\operatorname{Pr}\left(D_{i}=1 \mid x_{i}\right)=F\left(\beta x_{i}+\gamma u_{i}\right)
$$

Como já explicitado $D_{i}$ é igual a 1 se o indivíduo recebe o tratamento e 0 caso não receba; $x_{i}$ são as características observadas do indivíduo $i ; u_{i}$ corresponde à variável não observada e $\gamma$ representa o efeito de $u_{i}$ sobre a decisão de participação no programa. Se não existir viés de seleção, então $\gamma$ será igual a zero e a probabilidade de participação será exclusivamente determinada pelas características observáveis. Entretanto, na presença de viés de seleção, dois indivíduos com as mesmas co-variáveis observadas $x$ terão diferentes chances de receber tratamento.

Assumindo que dois indivíduos sejam pareados, $i$ e $j$, e que $F$ tenha uma distribuição logística, a odds dos indivíduos receberem tratamento é dada por:

$$
\frac{\pi_{i}}{1-\pi_{i}} \mathrm{e} \frac{\pi_{j}}{1-\pi_{j}}
$$

e a odds ratio é dada por:

$$
\frac{\frac{\pi_{i}}{1-\pi_{i}}}{\frac{\pi_{j}}{1-\pi_{j}}}=\frac{\pi_{i}\left(1-\pi_{j}\right)}{\pi_{j}\left(1-\pi_{i}\right)}=\frac{\exp \left(\beta x_{j}+\gamma u_{j}\right)}{\exp \left(\beta x_{i}+\gamma u_{i}\right)}=\exp \left[\gamma\left(u_{i}-u_{j}\right]\right.
$$

Se os indivíduos possuírem as mesmas características observáveis, então o vetor $x$ se cancela. Deste modo, se não houver diferenças nas variáveis não observadas $\left(u_{i}=u_{j}\right)$ e se estas variáveis não influenciarem a probabilidade de participação $(\gamma=0)$, a odds ratio será igual a 1 , implicando a não-existência de viés de seleção. Segue-se então, que se suas odds de participação diferirem - isto é, se a odds ratio for diferente de 1 - só pode ser devido à presença de não-observáveis. A análise de sensibilidade avalia o quanto do efeito do programa é alterado pela mudança nos valores de $\gamma$ e de $u_{i}-u_{j}$.

14 Hujer, Caliendo e Thonsen (2004). 
De acordo com Watson (2005), na prática isto significa examinar os limites da odds ratio de participação. Rosenbaum (2002) mostra que (11) implica os seguintes limites para a odds ratio):

$$
\frac{1}{e^{\gamma}} \leq \frac{\pi_{i}\left(1-\pi_{j}\right)}{\pi_{j}\left(1-\pi_{i}\right)} \leq e^{\gamma}
$$

Os indivíduos pareados possuem a mesma probabilidade de participação apenas se $e^{\gamma}$ $=1$. Entretanto, se $e^{\gamma}=2$, então indivíduos aparentemente similares em termos de $x$ irão diferir em suas probabilidades de receberem tratamento por um fator de até 2 . Assim, segundo Rosenbaum (2002), $e^{\gamma}$ seria uma medida do grau de ruptura a partir de um estudo livre de viés de seleção. ${ }^{15}$

\section{DESCRIÇÃO DOS DADOS E DAS VARIÁVEIS}

Os dados deste trabalho foram extraídos da Pesquisa de Orçamentos Familiares (POF) 2002-2003. A POF, realizada pelo Instituto Brasileiro de Geografia e Estatística (IBGE), é uma pesquisa amostral na qual são investigados os domicílios particulares permanentes, com representatividade por Unidade da Federação (UF). A coleta de dados da pesquisa foi realizada nas áreas urbanas e rurais de todo o território nacional no período de julho de 2002 a julho de 2003.

A amostra é constituída por 48.171 unidades de consumo (UC). De acordo com a POF, o conceito de unidade de consumo compreende um único morador ou conjunto de moradores que compartilham a mesma fonte de alimentação ou compartilham as despesas com moradia. Para efeito de divulgação de resultados, o termo família tem sido utilizado para representar o conceito de Unidade de Consumo.

Do total de famílias da amostra, 9,23\% delas são beneficiárias do programa BolsaEscola e a sua maioria concentra-se na região Nordeste e em áreas urbanas. De acordo com os microdados da POF, o valor mensal médio recebido pelas famílias participantes é de R\$31,68. Como a unidade de análise proposta neste trabalho é o conceito de família (unidade de consumo), foram consideradas como variáveis individuais as características do chefe, ao passo que as variáveis de despesas e rendimentos ${ }^{16}$ individuais foram agregadas por UC.

15 Apesar de este método não testar formalmente a hipótese CIA, fornece uma forma de julgar o quão grande deve ser a influência dos não-observáveis a fim de que os resultados do tratamento sejam alterados (ROSENBAUM, 2002, p.106 apud WATSON, 2005).

16 Foram considerados apenas os rendimentos monetários. 


\subsection{Variáveis Dependentes}

Além do efeito total sobre os gastos de consumo, será realizada também uma análise sobre os efeitos na sua composição com base nos valores absolutos. Pelo fato de o consumo de alimentos representar a maior proporção dos gastos das famílias participantes, a análise de seus componentes é particularmente importante. Através desta decomposição, pode-se, ainda, fazer algumas considerações a respeito dos efeitos sobre o status nutricional das famílias e de suas crianças. Deste modo, as seguintes variáveis serão analisadas.

QUADRO I - VARIÁVEIS DEPENDENTES - VALORES ABSOLUTOS

\begin{tabular}{|l|l|}
\hline Variáveis & \multicolumn{1}{c|}{ Descrição } \\
\hline Despesa Total & Somatório de todas as despesas \\
Despesas com Alimentos & $\begin{array}{l}\text { Despesas com a aquisição de alimentos tanto para consumo dentro } \\
\text { como fora do domićlio }\end{array}$ \\
Despesas com Habitação & $\begin{array}{l}\text { Estas despesas representam o somatório dos seguintes itens: } \\
\text { 1) Aluguel } \\
\text { 2) Serviços e Taxas } \\
\text { 3) Manutenção do Lar } \\
\text { 4) Eletrodomésticos } \\
\text { 5) Consertos de Artigos do Lar }\end{array}$ \\
\hline
\end{tabular}




\section{QUADRO 1 - VARIÁVEIS DEPENDENTES - VALORES ABSOLUTOS (CONTINUAÇÃO)}

\begin{tabular}{|c|c|}
\hline Variáveis & Descrição \\
\hline Despesas com Vestuário & Despesas com vestuário feminino, masculino e infantil. \\
\hline Despesas com Transportes & Despesas com transporte público e privado. \\
\hline $\begin{array}{l}\text { Despesas com produtos de Higiene } \\
\text { (cuidados pessoais) e Serviços Pessoais }\end{array}$ & $\begin{array}{l}\text { Despesas com a aquisição de artigos de higiene e beleza e serviços } \\
\text { pessoais, tais como: cabeleireiro, esteticista, consertos de artigos } \\
\text { pessoais etc. }\end{array}$ \\
\hline Despesas com Saúde & $\begin{array}{l}\text { Despesas com produtos e serviços relativos a assistência à saúde, } \\
\text { tais como consultas, remédios, plano de saúde etc. }\end{array}$ \\
\hline Despesas com Educação & $\begin{array}{l}\text { Neste tópico incluem-se despesas efetuadas com mensalidades, } \\
\text { livros didáticos, matrículas, uniformes escolares etc. }\end{array}$ \\
\hline Despesas com Recreação e Cultura & $\begin{array}{l}\text { Incluem-se despesas com brinquedos, diversões, esportes, livros e } \\
\text { revistas não-didáticos etc. }\end{array}$ \\
\hline Despesas com Fumo e Bebidas Alcoólicas & $\begin{array}{l}\text { Incluem-se despesas com cigarros, fumo e bebidas alcoólicas } \\
\text { consumidas dentro e fora do domicílio. }\end{array}$ \\
\hline Despesas Diversas & $\begin{array}{l}\text { Referem-se às despesas com jogos e apostas, cerimônias e festas } \\
\text { familiares, serviços profissionais etc. }\end{array}$ \\
\hline Outras Despesas Correntes & $\begin{array}{l}\text { Despesas com impostos, serviços bancários, pensões, previdência } \\
\text { privada etc. }\end{array}$ \\
\hline
\end{tabular}

\subsection{Variáveis Independentes}

Como visto, o modelo logit(probit) pelo qual o escore de propensão é calculado deve incluir variáveis preditoras que influenciam a participação no programa e os resultados de interesse. As variáveis utilizadas relacionam-se basicamente ao status de pobreza, uma vez que estas irão determinar tanto a elegibilidade para o programa como os efeitos sobre o consumo.

O escore de propensão estimado fornece a probabilidade predita de participação no programa de uma determinada família e através dos seus valores realiza-se o pareamento entre os grupos de tratamento e comparação. Deste modo, a inclusão de variáveis de região, domicílio, bem como as características do chefe têm por finalidade não 
só o cálculo da probabilidade de participação, mas também o pareamento das famílias em termos das características observáveis. Assim, o cálculo do ATT é realizado entre famílias que sejam de fato comparáveis, sendo esta comparabilidade alcançada através das variáveis incluídas no modelo de escore de propensão, tais como anos de estudo, raça, cor, características domiciliares etc. Estas variáveis representam, por sua vez, um controle para o cálculo do ATT. A hipótese adotada é que tais variáveis são ortogonais ao tratamento, dado que determinam a participação no programa, mas não são afetadas por este.

QUADRO 2 - VARIÁVEIS INDEPENDENTES

\begin{tabular}{|c|c|}
\hline Variáveis & Descrição \\
\hline rendapc & Renda mensal per capita (líquida das transferências do B.E) \\
\hline filhosUC2 & Número de filhos com idade menor ou igual a 15 anos \\
\hline anosest & Número de anos de estudos do chefe \\
\hline draca1 & Variável dummy para raça do chefe (branco $=1$, negro $=0$ ) \\
\hline dsexo1 & Variável dummy sexo do chefe (homem=1, mulher $=0$ ) \\
\hline Sul & Variável dummy de região \\
\hline Nordeste & Variável dummy de região \\
\hline Norte & Variável dummy de região \\
\hline Centrooeste & Variável dummy de região \\
\hline urb1 & Variável dummy para situação do domicílio (rural=1, urbana=0) \\
\hline metrop1 & Variável dummy para área metropolitana (metropolitana=1, não metropolitana=0) \\
\hline setor1 & Variável dummy para ocupação do chefe (formal=1, outros, incluindo desocupados=0) \\
\hline setor2 & Variável dummy para ocupação do chefe (informal=1, outros, incluindo desocupados=0) \\
\hline dcartcred & Variável dummy para cartão de crédito \\
\hline descoad1 & Variável dummy para escoadouro sanitário \\
\hline dabastag1 & Variável dummy para rede geral de abastecimento \\
\hline pavrua1 & Variável dummy para pavimentação na rua \\
\hline denergelet1 & Variável dummy para energia elétrica \\
\hline qtdebanhpp & Número de banheiros por pessoa na UC \\
\hline qtdecompp & Número de cômodos por pessoa na UC \\
\hline
\end{tabular}


Os resultados serão analisados em quatro seções. Na seção 5.1, serão desenvolvidas algumas consideraçôes a respeito do modelo probit construído para estimar os escores de propensão. Na seção 5.2 , serão examinados os resultados obtidos para as estimativas do efeito do tratamento. A sensibilidade das estimativas a pequenas mudanças na especificação do modelo de escore de propensão será analisada na seção 5.3. Finalmente, na seção 5.4, realiza-se uma análise de sensibilidade utilizando o método de Rosenbaum (2002), para as estimativas do efeito do tratamento sobre os resultados de interesse.

\subsection{O Modelo Probit}

O modelo probit é estimado a fim de predizer a probabilidade de participação no programa. Na geração dos modelos, deve-se considerar como grupo de comparação apenas os indivíduos elegíveis. Assim sendo, realizou-se um corte em relação à renda mensal per capita familiar para o valor de R $\$ 100,00$. Este corte tem por finalidade manter na amostra apenas os indivíduos do grupo de tratamento e controle que sejam de fato comparáveis. ${ }^{17}$

A Tabela 1 apresenta o resultado para o modelo de escore de propensão estimado. As especificações finais de tais modelos foram obtidas através de várias tentativas a fim de se chegar a especificações menos parcimoniosas e que satisfizessem a hipótese do "equilíbrio" (Balancing Hypothesis) entre as variáveis incluídas no modelo. A adoção de um modelo menos parcimonioso justifica-se pelo fato de que quanto maior o número de variáveis incluídas, melhor será o pareamento realizado, já que quanto maior o número de características observáveis utilizadas, mais similares serão os indivíduos dos grupos de tratamento e controle.

Através da análise dos preditores verifica-se que de fato alguns deles não são significativos, mas segundo Venetoklis (2004) isso não significa necessariamente que estas variáveis não devam ser incluídas no modelo. Rubin e Thomas (1996) (apud CALIENDO; KOPEINING, 2005) argumentam que uma variável só deve ser excluída da análise se houver um consenso de que esta não é apropriada (não relacionada com o resultado). De outra forma, é aconselhável incluí-la na estimativa do escore de propensão mesmo que não seja estatisticamente significativa. ${ }^{18}$ Os resultados obtidos

17 A renda per capita média mensal para a amostra inicial das famílias beneficiárias é cerca de R \$180,00 e de R \$ 640,00 para os não-participantes.

18 A introdução de variáveis não significativas não irá viesar as estimativas ou torná-las inconsistentes. (CALIENDO; KOPEINIG, 2005). 
por Zhao (2005) sugerem, ainda, que a inclusão de variáveis irrelevantes no modelo de escore de propensão não irão causar viés; no entanto, uma sobre especificação deste (e.g. a inclusão de termos não-lineares desnecessários) irá possivelmente viesar os resultados.

TABELA 1 - MODELO PROBIT

\begin{tabular}{lccrrrr}
\hline Variáveis & Coeficiente & $\begin{array}{c}\text { Erro Padrão } \\
\text { (Robusto) }\end{array}$ & $z$ & $P>|z|$ & Intervalo de Confiança de 95\% \\
\hline rendapc & $-0,0013$ & 0,0010 & $-1,27$ & 0,203 & $-0,0033$ & 0,0007 \\
filhosUC2 & 0,1961 & 0,0183 & 10,70 & 0,000 & 0,1601 & 0,2320 \\
anosest & $-0,0339$ & 0,0092 & $-3,69$ & 0,000 & $-0,0519$ & $-0,0159$ \\
dsexo1 & $-0,1079$ & 0,0611 & $-1,77$ & 0,077 & $-0,2276$ & 0,01188 \\
draca1 & $-0,0310$ & 0,0539 & $-0,58$ & 0,565 & $-0,1366$ & 0,0745 \\
Sul & 0,1721 & 0,1094 & 1,57 & 0,116 & $-0,0423$ & 0,3865 \\
Nordeste & 0,3857 & 0,0797 & 4,84 & 0,000 & 0,2294 & 0,5420 \\
Norte & $-0,0889$ & 0,0963 & $-0,92$ & 0,356 & $-0,2776$ & 0,0998 \\
Centrooeste & 0,0080 & 0,0983 & 0,08 & 0,935 & $-0,1846$ & 0,2006 \\
metrop1 & 0,3863 & 0,0770 & 5,02 & 0,000 & 0,2354 & 0,5371 \\
setor1 & $-0,0368$ & 0,0997 & $-0,37$ & 0,712 & $-0,2323$ & 0,1587 \\
setor2 & 0,1348 & 0,0954 & 1,41 & 0,158 & $-0,0522$ & 0,3219 \\
dcartcred1 & $-0,1909$ & 0,1203 & $-1,59$ & 0,113 & $-0,4268$ & 0,0450 \\
descoad1 & 0,1042 & 0,0766 & 1,36 & 0,174 & $-0,0459$ & 0,2544 \\
denergelet1 & 0,2578 & 0,0558 & 4,62 & 0,000 & 0,1484 & 0,3671 \\
pavrua1 & $-0,0150$ & 0,0523 & $-0,29$ & 0,773 & $-0,1175$ & 0,0874 \\
\hline cons & $-1,6646$ & 0,1592 & $-10,46$ & 0,000 & $-1,9766$ & $-1,3526$ \\
\hline
\end{tabular}

Fonte: Pesquisa de Orçamentos Familiares - POF/IBGE, 2002-2003. Microdados. Elaboração Própria.

TABELA 1.1. - LIMITE INFERIOR, NÚMERO DE TRATAMENTOS E NÚMERO DE CONTROLES PARA CADA BLOCO

\begin{tabular}{lrrr}
\hline $\begin{array}{l}\text { Limite inferior do Escore de Propensão } \\
\text { (com suporte comum) }\end{array}$ & Controle & Tratamento & Total \\
\hline 0,0470273 & & 40 & 401 \\
0,1 & 361 & 96 & 787 \\
0,15 & 691 & 199 & 1.055 \\
0,2 & 856 & 279 & 1.277 \\
0,25 & 998 & 344 & 1.144 \\
0,3 & 800 & 675 & 1.881 \\
0,4 & 1.206 & 400 & 866 \\
0,5 & 466 & 186 & 344 \\
0,6 & 158 & 120 & 215 \\
0,8 & 95 & 7 & 14 \\
\hline Total & 7 & 2.346 & 7.984 \\
\hline
\end{tabular}

Fonte: Pesquisa de Orçamentos Familiares - POF/IBGE, 2002-2003. Microdados. Elaboração Própria. 
De acordo com o modelo estimado, constata-se que em geral as variáveis de controle apresentam os sinais esperados. Observa-se que o número de filhos aumenta a probabilidade de participação no programa. $\mathrm{O}$ fato de o chefe ser do sexo masculino ou mais escolarizado diminui esta probabilidade. Este último fator está fortemente correlacionado com a renda, uma vez que normalmente os indivíduos mais escolarizados apresentam maiores rendimentos. Os indivíduos localizados na região Nordeste e em áreas não-metropolitanas apresentam maior probabilidade de serem elegíveis para o programa, o que pode se dever à forma de implementação do programa.

\subsection{O "Impacto" do Programa}

A análise será feita com base em três pontos: (a) a magnitude do valor estimado para o ATT ${ }^{19}$; (b) seu sinal e (c) sua significância estatística.

\subsubsection{Os Efeitos sobre a Despesa Total}

\section{TABELA 2 - CÁLCULO DO EFEITO DO TRATAMENTO PARA A DESPESA TOTAL}

\begin{tabular}{lc}
\hline Variável Dependente & Estimativa \\
\hline $\begin{array}{l}\text { Despesa Total } \\
\text { (Modelo Rendapc<=100) }\end{array}$ & 307,558 \\
$(3,037)^{*}$
\end{tabular}

Na Tabela 2 é reportada a estimativa do valor do efeito do tratamento para a despesa total. Observa-se que o valor médio dos gastos anuais para uma família beneficiária com renda mensal per capita de até $\mathrm{R} \$ 100,00$ é cerca $\mathrm{R} \$ 300,00$ superior à média dos gastos totais das famílias não-participantes. Considerando que o valor médio anual recebido por estas famílias é de R $\$ 347,53$, pode-se inferir que cerca de $85 \%$ deste valor é utilizado para gastos em geral.

\subsubsection{Os efeitos sobre os Componentes do Consumo}

O aumento dos gastos como conseqüência das transferências monetárias talvez não seja muito surpreendente; a questão a ser destacada, inclusive sob o ponto de vista

19 Os valores das despesas analisadas são anuais. 
da efetividade da política social, é o conhecimento de quais componentes apresentam maiores dispêndios. Segundo Attanasio e Mesnard (2005), a avaliação do programa será muito distinta se este resultar em um aumento nos gastos com educação do que se resultar em um aumento nos gastos com tabaco e bebidas alcoólicas.

A Tabela 3 fornece os valores do efeito médio do tratamento sobre os componentes do consumo. Esta tabela sugere que a maior parte das transferências recebidas é gasta em alimentação; neste caso, mais de $60 \%$ do valor das transferências é destinado à compra de alimentos. Pode-se também destacar que parte das transferências é destinada a despesas com vestuário, uma vez que os dados apontam que tais gastos são positivos e significativos. Verifica-se ainda que os gastos com produtos de higiene e serviços pessoais são superiores para as famílias beneficiárias, significando que uma parte das transferências é destinada à compra de artigos de higiene e beleza e serviços como cabeleireiro e consertos de artigos pessoais.

Em relação às despesas com itens relacionados à habitação, os dados sugerem que não há diferenças para estes gastos entre os grupos de tratamento e controle, já que os valores encontrados não são significativos. Do mesmo modo não se verifica maior incidência de gastos em itens de assistência à saúde.

Os gastos com educação, como esperado, apresentam valores positivos e significativos. Este resultado mostra que de fato uma parcela das transferências do Bolsa-Escola está sendo utilizada para as despesas com educação, o que pode ser atribuído à condicionalidade imposta pelo programa, reforçando positivamente o cumprimento desta.

Os resultados sugerem que as transferências recebidas não são alocadas para gastos com fumo e bebidas alcoólicas, dado que as famílias beneficiárias não parecem apresentar maiores níveis de gastos para estes itens. Em relação aos demais componentes, verifica-se que aparentemente os gastos com transportes, recreação e cultura, itens classificados como despesas diversas e gastos com aquisição de bens duráveis não são superiores quando comparados às despesas das famílias não-participantes. Do mesmo modo não se observaram maiores dispêndios para os itens incluídos na rubrica 'outras despesas correntes'. Pode-se concluir que dentre os diversos componentes analisados, os recursos recebidos do programa Bolsa-Escola são alocados principalmente para os gastos com alimentação, vestuário, educação e produtos de higiene e serviços pessoais; os demais não apresentaram distinções significativas entre os dois grupos.

Logo, os resultados indicam que a parcela das transferências que é gasta é quase que exclusivamente destinada ao aumento dos gastos de consumo, sugerindo que parte das transferências recebidas é de fato destinada para o aumento do consumo corrente. Esta ampliação, por sua vez, é inferior à unidade, dado que as famílias não despendem todo 
o incremento da renda, pois de acordo com resultados para a despesa total é provável que uma parcela das transferências seja destinada à poupança.

\section{TABELA 3 - CÁLCULO DO EFEITO DO TRATAMENTO PARA OS COMPO- NENTES DO CONSUMO}

\begin{tabular}{lc}
\hline Variáveis Dependentes & Estimativas \\
\hline Despesas com Alimentação & 225,772 \\
& $(4,370)^{*}$ \\
Despesas com Habitação & $-18,639$ \\
Despesas com Vestuário & $(-0,597)$ \\
& 33,823 \\
Despesas com Transportes & $(3,133)^{*}$ \\
Despesas com Higiene e Serviços Pessoais & 11,791 \\
& $(0,360)$ \\
Despesas com Assistência à Saúde ${ }^{20}$ & 23,120 \\
Despesas com Educação & $(3,090)^{\star}$ \\
Despesas com Recreação e Cultura & 9,796 \\
& $(0,774)$ \\
Despesas com Fumo e Bebidas Alcoólicas & 24,684 \\
Valor das Despesas com Despesas Diversas & $(7,755)^{*}$ \\
Despesas com Bens Duráveis & 3,351 \\
& $(0,986)$ \\
& $-6,478$ \\
& $(-1,409)$ \\
& $-6,846$ \\
& $(-0,535)$ \\
& 7,185 \\
& $(1,273)$ \\
& $-6,724$ \\
& $(-1,022)$ \\
\hline
\end{tabular}

Fonte: Pesquisa de Orçamentos Familiares - POF/IBGE, 2002-2003. Microdados. Elaboração Própria.

Notas: Valores das despesas em R\$, deflacionados e anualizados. Os valores entre parênteses representam a estatística t baseada no erro padrão calculado através do método de bootstrap (50 repetições). * Significativo a $1 \% .{ }^{*}$ Significativo a $5 \% .{ }^{* *}$ Significativo a $10 \%$.

20 Para o cálculo do efeito das despesas com saúde não foram considerados eventuais choques nos gastos uma vez que a POF não capta este tipo de efeito. No entanto, supõe-se que as diferenças para os choques na saúde não são significativas entre os grupos de tratamento e comparação. 


\subsubsection{Desagregando os Componentes de Alimentação}

Dada a importância dos gastos com alimentação, será analisado em mais detalhes o efeito do programa no consumo desse item. A Tabela 4 fornece o resultado para o itens de alimentação. Os dispêndios com alimentos foram desagregados nos seguintes subgrupos: (1) despesas com alimentos básicos (grãos, frutas, legumes, vegetais, farinhas, massas, pães, leite etc) e (2) despesas com alimentos não-básicos (alimentos processados, carnes, peixes, alimentos de origem animal etc).

A importância desta análise está no efeito indireto do programa sobre o status nutricional das famílias participantes. Como visto anteriormente, os dados sugerem que a maior parte dos recursos entregues aos beneficiários do Bolsa-Escola está sendo destinada a despesas com alimentos. Este acréscimo de recursos destinados à alimentação pode afetar a dieta das famílias de duas formas: primeiramente, isso pode fazer com que as famílias beneficiárias passem a consumir alguns alimentos que não eram consumidos antes por falta de renda (alimentos mais caros); segundo, o aumento de renda pode permitir que estas famílias aumentem a quantidade consumida de alimentos que já vinham sendo consumidos antes. (BRASIL, 2003).

\section{TABELA 4 - CÁLCULO DO EFEITO DO TRATAMENTO PARA OS COM- PONENTES DE ALIMENTAÇÃO: ALIMENTOS BÁSICOS E ALIMENTOS NÃO-BÁSICOS (VALORES ANUALIZADOS)}

\begin{tabular}{|c|c|}
\hline Variáveis Dependentes & Estimativas \\
\hline Grãos & $\begin{array}{l}90,557 \\
(4,737)^{*}\end{array}$ \\
\hline Frutas, legumes e vegetais & $\begin{array}{c}15,051 \\
(2,473)^{* \star}\end{array}$ \\
\hline Farinhas, massas e pães & $\begin{array}{c}20,154 \\
(2,377)^{\star *}\end{array}$ \\
\hline Leite & $\begin{array}{c}-21,584 \\
(-5,551)^{*}\end{array}$ \\
\hline Alimentos Processados & $\begin{array}{c}80,313 \\
(4,520)^{*}\end{array}$ \\
\hline Carnes e Peixes & $\begin{array}{c}40,205 \\
(3,264)^{*}\end{array}$ \\
\hline Alimentos de Origem Animal & $\begin{array}{c}2,823 \\
(1,150)\end{array}$ \\
\hline
\end{tabular}

Fonte: Pesquisa de Orçamentos Familiares - POF/IBGE, 2002-2003. Microdados. Elaboração Própria.

Notas: Para alimentos consumidos dentro de casa. Valores das despesas em R \$, deflacionados e anualizados. Os valores entre parênteses representam a estatística $t$ baseada no erro padrão calculado através do método de bootstrap (50 repetiçóes). ${ }^{*}$ Significativo a $1 \%$. ** Significativo a 5\%. ** Significativo a $10 \%$. 
A Tabela 4 mostra que as despesas dos beneficiários do B.E são superiores para o consumo de alimentos básicos e não-básicos - o que pode sugerir efeitos positivos sobre a qualidade e diversificação da dieta devido ao aumento do consumo de ambas as classes de alimentos. De acordo com os resultados, uma parcela significativa dos gastos com alimentação é destinada principalmente à aquisição de produtos como grãos, alimentos processados, carnes e peixes. Esses resultados são importantes na medida em que a melhoria na quantidade e na diversificação da dieta pode trazer benefícios à saúde $\mathrm{e}$ conseqüentemente à produtividade dos adultos bem como à saúde e ao bem-estar das crianças possibilitando, assim, o acúmulo de capital humano.

\subsection{Sensibilidade à Especificação do Escore de Propensão}

Nesta seção será verificada a sensibilidade da estimação dos efeitos do tratamento a pequenas mudanças na especificação do modelo de escore de propensão, como por exemplo a inclusão ou retirada de termos de ordem superior. No caso de o escore de propensão ser estimado parametricamente, torna-se uma importante questão o quão sensíveis são as estimativas do efeito de tratamento às especificações do modelo. (ZHAO, 2005).

Se os resultados obtidos com a nova especificação forem similares aos alcançados através do modelo original, pode-se considerar que as estimativas são robustas a pequenas mudanças na especificação do modelo. Segundo Dehejia (2005), se no entanto, os resultados forem altamente sensíveis a mudanças na especificação, tal método pode tornar-se inválido. Ainda de acordo com o autor, mesmo na falta de um experimento aleatório, deve-se hesitar em adotar estimativas que sejam sensíveis à especificação do escore de propensão.

Os resultados apresentados nas Tabelas 5 e 6 demonstram que as estimativas do efeito do tratamento não são particularmente sensíveis à especificação utilizada no modelo de escore de propensão, uma vez que os resultados obtidos são semelhantes aos apresentados na Tabela 3. Deste modo, pode-se concluir que os resultados são robustos a pequenas mudanças na especificação do modelo e que o emprego de tal método é viável. 


\section{TABELA 5 - CÁLCULO DO EFEITO DO TRATAMENTO UTILIZANDO A NOVA ESPECIFICAÇÃO DO MODELO DE ESCORE DE PROPENSÃO}

\begin{tabular}{lc}
\hline Variáveis Dependentes & Estimativas \\
\hline Despesas com Alimentação & 224,134 \\
& $(5,241)^{\star}$ \\
Despesas com Habitação & $-19,391$ \\
& $(-0,636)$ \\
Despesas com Vestuário & 34,640 \\
& $(3,382)^{\star}$ \\
Despesas com Transportes & 8,160 \\
& $(0,269)$ \\
Despesas com Higiene e Serviços Pessoais & 23,398 \\
Despesas com Assistência à Saúde & $(3,315)^{\star}$ \\
Despesas com Educação & 8,863 \\
& $(0,781)$ \\
Despesas com Recreação e Cultura & 24,338 \\
Vespesas com Fumo e Bebidas Alcoólicas & $(7,125)^{\star}$ \\
Valor das Despesas com Despesas Diversas & 3,223 \\
& $(1,113)$ \\
& $-6,623$ \\
& $(-1,242)$ \\
& -7.202 \\
& $(-0.497)$ \\
& 6,620 \\
& $(1,211)$ \\
& $-7,410$ \\
& $(-0.958)$ \\
\hline
\end{tabular}

Fonte: Pesquisa de Orçamentos Familiares - POF/IBGE, 2002-2003. Microdados. Elaboração Própria.

Notas: A nova especificação exclui a variável dummy de sexo. Valores das despesas em R\$, deflacionados e anualizados. Os valores entre parênteses representam a estatística $t$ baseada no erro padrão calculado através do método de bootstrap (50 repetições). * Significativo a 1\%. ** Significativo a 5\%. *** Significativo a $10 \%$. 


\section{TABELA 6 - CÁLCULO DO EFEITO DO TRATAMENTO UTILIZANDO A NOVA ESPECIFICAÇÃO DO MODELO DE ESCORE DE PRO- PENSÃO}

\begin{tabular}{lc}
\hline Variáveis Dependentes & Estimativas \\
\hline Despesas com Alimentação & 229,719 \\
& $(4,461)^{\star}$ \\
Despesas com Habitação & $-19,289$ \\
& $(-0,587)$ \\
Despesas com Vestuário & 34,942 \\
& $(3,038)^{\star}$ \\
Despesas com Transportes & 13,783 \\
& $(0,366)$ \\
Despesas com Higiene e Serviços Pessoais & 22,682 \\
& $(3,903)^{\star}$ \\
Despesas com Assistência à Saúde & 12,206 \\
Despesas com Educação & 12,206 \\
& $(1,113)$ \\
Despesas com Recreação e Cultura & 23,592 \\
& $(7,109)^{*}$ \\
Despesas com Fumo e Bebidas Alcoólicas & 0,902 \\
Valor das Despesas com Despesas Diversas & $(0,265)$ \\
& $-7,295$ \\
\hline
\end{tabular}

Fonte: Pesquisa de Orçamentos Familiares - POF/IBGE, 2002-2003. Microdados. Elaboração Própria.

Notas: A nova especificação exclui a variável dummy para região Metropolitana. Valores das despesas em $\mathrm{R} \$$, deflacionados e anualizados. Os valores entre parênteses representam a estatística $t$ baseada no erro padrão calculado através do método de bootstrap (50 repetições). ${ }^{*}$ Significativo a $1 \% . * *$ Significativo a $5 \% .{ }^{* *}$ Significativo a $10 \%$.

\subsection{Análise de Sensibilidade}

A análise de sensibilidade questiona o "quanto" de viés de seleção deverá estar presente a fim de tornar aceitável a hipótese nula de que não há efeito de tratamento. Segundo Diprete e Gangl (2004), a metodologia empregada inicia-se com a estimativa do ATT utilizando métodos de pareamento (o escore de propensão), baseada na hipótese de 
independência condicional. No passo seguinte, postula-se a existência de uma variável omitida $Z$, que afeta a probabilidade de participação no programa (i.e., a probabilidade de $D=1$ ), condicional em $X$. Conforme o impacto potencial de $Z$ sobre $D$ (expresso em termos dos limites das odds ratio) torna-se mais forte, o intervalo de confiança dos efeitos estimados amplia-se, e o nível de significância do teste da hipótese nula, de que não há efeito de $D$ sobre $Y$, aumenta (i.e, o p-valor eleva-se).

Os resultados da Tabela 7 mostram que a robustez ao viés de seleção varia entre as variáveis de resultado. Em geral, o efeito do tratamento parece ser robusto a uma possível presença de viés de seleção, pois quanto maior o valor do nível crítico $\Gamma$, mais robusta a variável se apresenta.

Para a variável alimentação, o nível crítico de $\Gamma$ é de 1,15 , significando que se a presença de variáveis não observadas levarem a uma diferença na odds ratio de receber tratamento entre os indivíduos dos grupos de tratamento e controle por um fator de 1,15 , então pode-se questionar o efeito positivo das transferências do programa sobre os gastos com alimentação. Os resultados sugerem que as despesas com vestuário e educação também parecem ser robustas à presença de viés de seleção. No caso da variável educação, o nível crítico de $\Gamma$ é de 1,6 . No entanto, variáveis como despesas com habitação, transportes, saúde, fumo e bebidas alcoólicas aparentam ser menos robustas à presença de não-observáveis, dado que apresentam um valor crítico mais próximo da unidade. Segundo Diprete e Gangl (2004) é importante notar que estes resultados são considerados como os piores cenários. Cabe ressaltar que o valor de 1,10 , por exemplo, não sugere necessariamente que não exista um efeito positivo sobre as despesas totais. De acordo com os autores, isso implica apenas que o intervalo de confiança para o efeito do tratamento irá incluir zero se a presença de viés de seleção levar a odds de participação a diferir entre os grupos de tratamento e controle por um fator de 1,10 . No caso de uma variável omitida possuir forte influência sobre a participação no programa, mas apenas uma pequena influência sobre a variável de resultado, o intervalo de confiança não irá conter zero. Apesar de a análise de sensibilidade de Rosenbaum apresentar os resultados para o pior cenário, ele demonstra quão grande deve ser a influência de uma variável não observada a fim de questionar as conclusões obtidas através de métodos de pareamento. 
TABELA 7 - ANÁLISE DE SENSIBILIDADE (ROSENBAUM BOUNDS) PARA DESPESAS TOTAIS E SEUS COMPONENTES

\begin{tabular}{|c|c|c|c|c|c|}
\hline Variáveis & $\Gamma$ & p-crítico & Variáveis & $\Gamma$ & p-crítico \\
\hline Despesa Total & $\begin{array}{r}1 \\
1,05 \\
1,10\end{array}$ & $\begin{array}{l}<0,0001 \\
0,003019 \\
0,19941\end{array}$ & $\begin{array}{l}\text { Recreação e } \\
\text { Cultura }\end{array}$ & $\begin{array}{r}1 \\
1,05 \\
1,10\end{array}$ & $\begin{array}{l}0,001698 \\
0,017532 \\
0,092536\end{array}$ \\
\hline Alimentação & $\begin{array}{r}1 \\
1,05 \\
1,10 \\
1,15\end{array}$ & $\begin{array}{l}<0,0001 \\
0,001497 \\
0,022996 \\
0,143014\end{array}$ & $\begin{array}{l}\text { Fumo e Bebidas } \\
\text { Alcoólicas }\end{array}$ & $\begin{array}{r}1 \\
1,05\end{array}$ & $\begin{array}{l}0,576545 \\
0,252507\end{array}$ \\
\hline Habitação & $\begin{array}{r}1 \\
1,05\end{array}$ & $\begin{array}{l}0,718162 \\
0,327901\end{array}$ & $\begin{array}{l}\text { Despesas } \\
\text { Diversas }\end{array}$ & $\begin{array}{r}1 \\
1,05\end{array}$ & $\begin{array}{l}0,566189 \\
0,861275\end{array}$ \\
\hline Vestuário & $\begin{array}{r}1 \\
1,05 \\
1,10 \\
1,15\end{array}$ & $\begin{array}{l}<0,0001 \\
0,00348 \\
0,042171 \\
0,212596\end{array}$ & $\begin{array}{l}\text { Despesas } \\
\text { Correntes }\end{array}$ & $\begin{array}{r}1 \\
1,05 \\
1,10 \\
1,15 \\
1,20\end{array}$ & $\begin{array}{l}<0,0001 \\
<0,0001 \\
0,001405 \\
0,013732 \\
0,072655\end{array}$ \\
\hline Transportes & $\begin{array}{r}1 \\
1,05\end{array}$ & $\begin{array}{l}0,967944 \\
0,800071\end{array}$ & $\begin{array}{l}\text { Despesas com } \\
\text { Bens Duráveis }\end{array}$ & $\begin{array}{r}1 \\
1,05\end{array}$ & $\begin{array}{l}0,156628 \\
0,002404\end{array}$ \\
\hline $\begin{array}{l}\text { Higiene e Serviços } \\
\text { Pessoais }\end{array}$ & $\begin{array}{r}1 \\
1,05 \\
1,10\end{array}$ & $\begin{array}{l}<0,0001 \\
0,009136 \\
0,082709\end{array}$ & & & \\
\hline Saúde & $\begin{array}{r}1 \\
1,05\end{array}$ & $\begin{array}{l}0,121935 \\
0,413102\end{array}$ & & & \\
\hline Educação & $\begin{array}{r}1 \\
1,05 \\
1,10 \\
1,15 \\
1,20 \\
1,25 \\
1,30 \\
1,40 \\
1,50 \\
1,60\end{array}$ & $\begin{array}{l}0 \\
0 \\
0 \\
<0,0001 \\
<0,0001 \\
<0,0001 \\
<0,0001 \\
<0,0001 \\
0,006715 \\
0,120755\end{array}$ & & & \\
\hline
\end{tabular}

Fonte: Pesquisa de Orçamentos Familiares - POF/IBGE, 2002-2003. Microdados. Elaboração Própria.

Nota: A segunda coluna contém os valores de $\mathrm{p}+$ para os limites de Rosenbaum. Se $\Gamma=e^{\gamma}=1$ implica a não-existência de viés devido a não-observáveis. 


\section{CONSIDERAÇOEES FINAIS}

Este trabalho pretendeu analisar os impactos das transferências monetárias advindas de programas sociais de transferências de renda sobre os gastos das famílias beneficiárias. Para isso, foram utilizados dados referentes ao programa Bolsa-Escola (B.E), tomando como um exemplo representativo de programas de transferência condicional de renda. Buscou-se mensurar os efeitos das transferências do B.E sobre os gastos totais e sobre seus componentes, para as famílias beneficiárias e, deste modo, sobre o bem-estar destas e de suas crianças, visando preencher uma lacuna deste tipo de estudo para o Brasil.

Programas de transferência condicional de renda, como o B.E, apresentam como objetivo a redução dos níveis de desigualdade e pobreza corrente através da transferência de renda para as famílias pobres e a provisão de incentivos ao combate à pobreza futura, através do aumento da freqüência escolar entre as crianças participantes e, portanto, do acúmulo de capital humano. Tais políticas, no entanto, apresentam também efeitos "indiretos", relacionados à melhoria do bem-estar das famílias. Estudos recentes ${ }^{21}$ evidenciam os potenciais efeitos dos programas de transferência sobre a redução das desigualdades e pobreza no País, destacando a importância deste tipo de política.

A restrição imposta pelos dados fez com que optássemos pelo uso do método de escore de propensão, utilizando informações coletadas em apenas um ponto no tempo. A disponibilidade de dados em dois pontos no tempo permitiria a utilização do método da diferença em diferenças, que produz melhores resultados quando comparados aos estimadores cross-section, uma vez que é mais robusto à presença de viés de seleção. Dada a restrição de dados, a utilização do pareamento baseado no escore de propensão parece ser a metodologia mais indicada, pois tal método permite parear os indivíduos do grupo de tratamento e controle através das características observáveis, reduzindo assim o viés. Ressalta-se, também, a importância da utilização de métodos de análise de sensibilidade visando concluir o emprego desta metodologia.

De acordo com os resultados obtidos, o valor médio dos gastos anuais para uma família beneficiária com renda mensal per capita de até R \$ 100,00 é cerca R \$300,00 superior à média dos gastos totais das famílias não-participantes. Considerando que o valor médio anual recebido por estas famílias é de $\mathrm{R} \$ 347,53$, pode-se inferir que cerca de $85 \%$ deste valor é utilizado para gastos em geral.

Os resultados estimados indicam um efeito positivo das transferências monetárias sobre o consumo das famílias pobres beneficiárias. O fato de os recursos serem prio-

21 Rocha (2004); Soares (2006); Ferreira, Leite e Litchfield (2006). 
ritariamente destinados a despesas com alimentos, educação, produtos de higiene e vestuário em detrimento de itens como bebidas e cigarros, despesas diversas e bens duráveis significa que, em alguma medida, estes recursos estão sendo gastos de forma "eficiente". Além disso, é bastante provável que o aumento do consumo destas famílias eleva o seu nível de bem-estar, representando um "alívio" imediato sobre a pobreza. Deve-se ressaltar que o aumento do consumo para os itens especificados ameniza as adversidades destas famílias no curto prazo, mas também pode proporcionar efeitos no longo prazo.

Os resultados do trabalho indicam que os gastos das famílias beneficiárias são destinados principalmente à melhoria da dieta das famílias (quantidade e diversificação) e conseqüentemente de suas crianças e à obtenção de itens relacionados à educação infantil, higiene e saúde. Tais resultados podem sugerir que programas de transferência de renda, como o caso do Bolsa-Família, que transfere um valor monetário superior, podem também ter efeitos de longo prazo uma vez que a melhoria do status nutricional, o incentivo à educação e os cuidados com a saúde irão permitir o acúmulo de capital humano destas crianças e, deste modo, permitirão a quebra do ciclo de pobreza destas famílias.

A relevância das avaliações de impacto é direta, pois seus efeitos podem ser associados a programas sociais ou melhorias em programas existentes para atingir os objetivos da política social. Por meio dos estudos de avaliação podem ser analisados os impactos dos programas condicionais de transferência de renda no curto, no médio e no longo prazo sobre as variáveis de interesse, e através disto fazer inferências a respeito de sua eficácia no combate à pobreza e à desigualdade.

O programa Bolsa-Família, criado em 2003, destaca-se como um dos maiores programas de transferência de renda existentes no País. Este programa apresenta uma vasta cobertura em relação à proporção de famílias pobres e apresenta, também, valores superiores para as transferências monetárias. Neste sentido, o B.F apresenta implicações políticas e sociais muito maiores em relação ao B.E, que reforçam a grande importância de se realizar avaliações de impactos. A sugestão de uma agenda de pesquisa envolveria, portanto, a análise do B.F. em todas as possíveis esferas de impactos, ou seja, não só analisando o cumprimento de suas condicionalidades, mas também os seus efeitos não explícitos, destacando-se os efeitos das transferências monetárias sobre os gastos de consumo das famílias. Programas com formatos bastante similares ao B.F., como o Progresa (México) e FA (Colômbia), apresentam avaliações em relação a este item. A importância de se proceder a uma análise completa destes programas tem por objetivo a mensuração de seus impactos sobre a pobreza a partir de diversos aspectos. A análise sobre o consumo das famílias torna-se particularmente importante na medida em que seu aumento e diversidade contribuem para a construção de uma 
"infra-estrutura" doméstica, que, por sua vez, permite o cumprimento das condicionalidades. E assim, considerados de forma abrangente, tais programas de transferência de renda teriam a capacidade de promover a inclusão social, contribuindo para a emancipação das famílias beneficiárias e para a construção de meios e condições para que estas possam sair da situação de vulnerabilidade em que se encontram.

\section{REFERENCIAS}

ATTANAZIO, O. et al. How effective are conditional cash transfers? evidence from Colombia. London: The Institute for Fiscal Studies, 2005. (Briefing Note, n. 54) Disponível em: < http://www.ifs.org.uk/publications.php>. Acesso em: $12 / 11 / 2006$.

ATTANAZIO, O.; MEGHIR, C.; VERA-HERNANDEZ, M. et al. Baseline report on the evaluation of familias en acción. London: The Institute for Fiscal Studies, 2004. Disponível em: < http://www.ifs.org.uk/publications.php>. Acesso em: $12 / 11 / 2006$.

ATTANAZIO, O., MESNARD, A. The impact of a conditional cash transfer programme on consumption in Colombia. London: The Institute for Fiscal Studies, 2005. Disponível em: < http://www.ifs.org.uk/publications.php>. Acesso em: $12 / 11 / 2006$.

BECKER S.O.; ICHINO, A. Estimation of average treatment effects based on propensity score. Stata Journal, v. 2, n. 4, p. 358-377, 2002. Disponível em: $<$ http://www.sobecker.de/pscore.html.>. Acesso em: 12/11/2006.

BRASIL. Ministério da Saúde. Avaliação do programa Bolsa-Alimentação. Brasília, DF: Ministério da Saúde, 2003. (Estudo 02: Análise de impacto preliminar)

CALIENDO, M.; KOPEINIG, S. Some practical guidance for the implementation of propensity score matching. Bonn, Germany: Institute for the Study of Labor (IZA), 2005. (IZA Discussion Papers, 1588)

CAMERON, A. C.; TRIVEDI, P. K. Microeconometrics: methods and applications. New York: Cambridge University Press, 2005.

DAS, J.; DO, Q.; ÖZLER, B. Conditional Cash Transfer and the Equity-Efficiency Debate. World Bank, Research working paper, WPS 3280, 2004

DEHEJIA, R. Practical propensity score matching: a reply to Smith and Todd. Journal of Econometrics, v. 125, n. 1-2, p. 355-364, 2005.

DIPRETE, T.; GANGL, M. Assessing bias in the estimation of causal effects: Rosenbaum bounds on matching estimators and instrumental variables estimation with imperfect instruments. Sociological Methodology, v. 34, n. 1, p. 271-310, Abr. 2004. Disponível em: < http://www.wjh.harvard.edu/ winship/cfa_papers/ HBprop_021204.pdf>. Acesso em: 12/11/2006. 
FERREIRA, F.; LEITE, P.; LITCHFIELD, J. The rise and fall of Brazilian inequality: 1981-2004. Washington, D.C.: World Bank, 2006. (World Bank Policy Research Working Paper, 3867)

HECKMAN, J.; ICHIMURA, H.; TODD, P. Matching as an econometric evaluation estimator: evidence from evaluating a job training program. Review of Economic Studies, v. 64(4), n. 221, p. 605-654, Oct. 1997.

HECKMAN, J.; LALONDE, R.; SMITH, J. The economics and econometrics of active labor market programs. In: ASHENFELTER, O., CARD, D. (Eds.) The Handbook of Labor Economics. Amsterdam: North Holland, v. 3A, part. 6, cap. 31, p. 1865-2097, 1999.

HIRANO, K.; IMBENS, G. W.; RIDDER, G. Efficient estimation of average treatment effects using the estimated propensity score. Cambridge, MA.: National Bureau of Economic Research, 2000. (NBER Technical Working Paper, n.251). Disponível em: <http://ideas.repec.org/s/nbr/nberwo.html>. Acesso em: 04/01/2006.

HUJER, R.; CALIENDO, M.; THOMSEN, S. New evidence on the effects of job creation schemes is Germany: a matching approach with threefold heterogeneity. Bonn, Germany: IZA, 2004. (Working Paper, 750)

IMBENS, G.W. The role of propensity score in estimating dose: response functions. Biometrika, v. 87, n. 3, p.706-710, 2000.

RESENDE, A.C.C. Avaliando resultados de um programa de transferência de renda: O impacto do Bolsa-Escola sobre os gastos das familias brasileiras. 2006. Dissertação (Mestrado), CEDEPLAR/UFMG.

RAWLINGS, L. B.; RUBIO, G. M. Evaluating the impact of conditional cash transfer programs: lessons form Latin America. Washington, DC: The World Bank, 2003. Disponível em: <World Bank Policy Research Working Paper 3119>. Acesso em: 05/05/2006.

ROCHA, S. Impacto sobre a pobreza dos novos programas federais de transferência de renda. Revista Economia Contemporánea, v. 9, n. 1, p. 153-185, jan./abr. 2005.

. Pobreza no Brasil: o que mudou nos últimos 30 anos? Rio de Janeiro: INAE - Instituto Nacional de Altos Estudos, 2004. (Estudos e pesquisas, n. 83)

ROSENBAUM, P. Observational Studies, Springer, New York. 2002.

;- RUBIN, D. B. The Central role of the propensity score in observational studies for causal effects. Biometrika, v. 70, n. 1, p. 41-55, 1983.

SOARES, S. Distribuição de renda no Brasil de 1976 a 2004 com ênfase no período entre 2001 e 2004. Brasília: IPEA, 2006. (Texto para discussão, n.1 166) Disponível em: < http://www.ipea.gov.br/pub/td/2006/td_1166.pdf>. Acesso em: 30/11/2006. 
VAZ, B. L. O.; ASSUNÇÃO, J. J. Efeito do programa Bolsa-Escola sobre as despesas das familias. 2006. Dissertação (Mestrado), PUC-Rio.

VENETOKLIS, T. An Evaluation of wage subsidy programs to SMEs utilizing propensity score matching. Helsinki: Government Institute for Economic Research, 2004. VATT Research Reports, 104. Disponível em: < http://en.vatt.fi/file/vatt_publication_pdf/t106.pdf $>$. Acesso em: 12/11/2006.

WATSON, I. The earnings of casual employees: the problem of unobservables. Paper presented to HILDA Survey Research Conference, University of Melbourne, 28-29 September 2005.

ZHAO, Z. Sensitivity of propensity score methods to the specifications. Bonn, Germany Institute for the Study of Labor (IZA), 2005. (IZA Discussion Papers, 1873). 